\title{
PAPER
}

\section{Cognitive impairment in probable multiple sclerosis}

\section{A Achiron, Y Barak}

See end of article for authors' affiliations .....................

Correspondence to: Dr A Achiron, Multiple Sclerosis Center, Sheba Medical Center, Tel Hashomer, 52621, Israel: achiron@post.tau.ac.il

Received 19 June 2002 In revised form 9 October 2002 Accepted 13 December 2002

\begin{abstract}
Objectives: To evaluate and characterise cognitive impairment in the very early stage of multiple sclerosis (MS), in which patients are still diagnosed as suffering from probable MS.

Methods: The Brief Repeatable Battery-Neuropsychological (BRB-N) that has been validated for MS patients was used. Abnormal performance was defined as one standard deviation below the mean reported for healthy age matched subjects. Neurological disability and brain magnetic resonance imaging (MRI) were performed for all patients. Correlation coefficients were calculated between disease burden variables and performance on the BRB-N.

Results: Sixty seven patients with probable MS were evaluated within a mean of one month of the onset of new neurological symptoms. Evidence for the presence of cognitive impairment was shown in $53.7 \%$ of patients. Verbal abilities and attention span were most frequently affected. Impairment was not correlated with neurological disability or MRI disease burden.

Conclusion: Prevalent cognitive impairment already exists at onset of MS.
\end{abstract}

$\mathrm{P}$ robable multiple sclerosis (MS) is defined by the first appearance of various combinations of motor, sensory, coordination, visual, and cognitive impairments, as well as symptoms of fatigue and urinary tract dysfunction compatible with central nervous system (CNS) involvement in young adults. ${ }^{1}$ Despite the widespread use of electrophysiological studies, brain and spinal cord imaging, and immunological parameters that contribute to diagnosis and better understanding of the early processes involved in $\mathrm{MS}^{2{ }^{2}}$ there are still aspects that require further investigation. Cognitive impairment in probable MS has been described sparsely. ${ }^{4}$ Growing awareness and accumulating data regarding the cognitive impairment and its progression in MS patients has received an important place in neurological research in the past decade. ${ }^{5}$ More specifically, memory loss was reported to be prevalent early in the natural history of $\mathrm{MS} .{ }^{7}$ Coupled with studies showing tissue abnormalities and destruction, axonal loss, and brain atrophy early in the disease course, ${ }^{8}$ it is imperative to acquire a body of knowledge made up of histological, physiological, imaging, and clinical parameters pertaining to probable MS. Such knowledge may contribute to better delineation of baseline cognitive impairment so that interventions designed to limit or delay tissue damage may be initiated.' These may enable prevention of the irreversible consequences of forgoing treatment such as changes in brain volume, ${ }^{10}$ and loss of axonal integrity. ${ }^{11}$ The diagnosis of definite MS requires a second neurological exacerbation. ${ }^{12}$ Between the first and second relapse, patients are classified as suffering from probable MS. This may in fact be a critical period within which pathological processes involved in cognitive impairment are not dormant, similar to magnetic resonance imaging (MRI) studies showing activity of the disease while physicians await clinical confirmation.

The aim of the present study was to evaluate the frequency of cognitive impairment in patients diagnosed as suffering from probable MS, and correlate these findings with demographic, clinical, and brain MRI parameters.

\section{SUBJECTS AND METHODS} Study participants

Patients evaluated at the Multiple Sclerosis Center, Sheba Medical Center, Tel Hashomer, Israel, aged 15-55 years (inclusive), who had onset of new neurological symptoms within the three months preceding the study were recruited. Patients had to have experienced symptoms reflecting predominantly white matter involvement, occurring for a period of at least 48 hours and resolving partially or completely within 2-8 weeks (that is, attack), with clinical evidence of at least one lesion producing objective CNS dysfunction, and with paraclinical evidence of demyelinating lesions by brain MRI examination (positive brain MRI) (CPMS C2 or CPMS C 3 ). ${ }^{12}$

Positive brain MRI was defined in accordance with Fazekas and colleagues' criteria $^{13}$ as the appearance of three or more ellipsoid demyelinating lesions whose diameter is $3 \mathrm{~mm}$ or more, within the brain. Of these lesions at least one was evident within the periventricular white matter or brainstem in a diameter of $6 \mathrm{~mm}$ or longer.

\section{Neuropsychological assessment}

The neuropsychological tests battery was based on the Brief Repeatable Battery- Neuropsychological (BRB-N). ${ }^{14} 15$

In brief, the BRB- $\mathrm{N}$ is composed of the following tests. (1) The selective reminding test measures verbal learning and delayed recall through presentation of a list of 12 words and five subsequent learning trials. Delayed recall (SRTD) is assessed after an 11 minute delay. (2) The 10/36 spatial recall test (SPART) assesses visual learning and recall by recreating the pattern of 10 checkers on a $6 \times 6$ checkerboard viewed for 10 seconds. (3) The symbol digit modalities test (SDMT) measures complex attention and concentration by requiring the subject to associate symbols with numbers and quickly generate the number when shown the symbol during 90 seconds. (4) The paced auditory serial addition task (PASAT) evaluates sustained attention and information processing speed, and is measured by asking the patient to add each number to the one immediately preceding it while numbers are presented every three seconds. (5) The word list generation (WLG) measures semantic verbal fluency, evaluating the spontaneous production of words beginning with a particular letter during 60 seconds.

Abbreviations: BRB-N, Brief Repeatable Battery-Neuropsychological; CDT, clock drawing test; CNS, central nervous system; MRI, magnetic resonance imaging; MS, multiple sclerosis; PASAT, paced auditory serial addition task; SDMT, symbol digit modalities test; SPART, spatial recall test; SRTD, selective reminding test; WLG, word list generation 
The BRB-N (translated and validated in Hebrew) ${ }^{16}$ was administered in a single 45 minute testing session by a trained clinical and rehabilitation psychologist experienced in administration of these tests. Scoring of all BRB-N measures was verified by our centre's psychiatrist (YB) before data entry. Normative values were adopted in accordance with values obtained by Boringa and colleagues, ${ }^{15}$ and abnormal values were defined as one standard deviation (SD) below the mean for normal controls. In addition, the clock drawing test (CDT) was administered in accordance with the procedure described by Shulman and colleagues. ${ }^{17}$ The CDT encompasses a range of higher level cognitive abilities, including integrative functions, abstract thinking, and visuospatial organisation. ${ }^{18}$

\section{Disability assessment}

Neurological disability was assessed by the Exapanded Disability Status Scale (EDSS). ${ }^{19}$ The EDSS is an eight functional system scale that includes: motor, sensory, cerebellar, brain stem, visual, mental, sphincteric, and others. Each is graded from $0=$ no disability, to 5 or $6=$ maximal disability. According to the score in each functional system, an integrated score between $0=$ normal examination and $10=$ death from MS, is derived.

\section{Psychiatric assessment}

Each patient underwent a clinical interview that was semistructured and is the modified Hebrew version of the specific interview originally designed for use with the Brief Psychiatric Rating Scale. In addition, the Goldberg anxiety and depression scale was used to assess the probability of the presence of a clinically relevant disorder as described previously. ${ }^{20}$

\section{Brain MRI}

Brain MRI examinations were obtained using a 2.0 Tesla Imager (Elscint, Israel). For each MRI examination the following data were acquired: (1) sagittal Tl weighted localiser images (500/12 TR/TE), $24 \mathrm{~cm}$ field of view, $256 \times 180$ matrix; (2) axial dual spin-echo (PD) and T2 weighted sequence (5500/16-128 TR/TEs), $22 \mathrm{~cm}$ field of view, 256×256 matrix; and (3) axial Tl weighted images (500/12 TR/TE), 24 $\mathrm{cm}$ field of view, 256×180 matrix before and after intravenous administration of $0.1 \mathrm{mmol} / \mathrm{kg}$ of Gd-DTPA. All examinations covered the brain from the level of the foramen magnum to the higher convexity of the scalp, using $3 \mathrm{~mm}$ slice thickness and no interslice gap, yielding 44 contiguous images. Disease burden was quantified using the multispectral MS Analyze software which includes several algorithms that enable the integration of data from at least three MR pulse sequences. Automatic detection and quantification of white matter lesions on MR images was thus obtained. ${ }^{21}$

\section{Statistical analysis}

Procedures for evaluating BRB-N performance as well as the composite domains were defined in accordance with normative values reported by Boringa and colleagues ${ }^{15}$; selection of variables for the composite cognitive domains analysis was guided by the factor analysis reported by Fischer and colleagues. $^{22}$ The correlations between the demographic parameters and the cognitive subgroups were tested using Fisher's exact test and Pearson's $\chi^{2}$ test for the categorical parameters, and ANOVA for continuous parameters. Differences between the normal and abnormal results in each of the composite cognitive domains and EDSS and MRI parameters were tested using the Mann-Whitney $U$ test for independent groups. Data are presented as mean $(\mathrm{SD}) ; \mathrm{p}<0.05$ was considered statistically significant.
Table 1 Neuropsychological tests in patients with probable multiple sclerosis

\begin{tabular}{lll}
\hline Neuropsychological test & No. patients & \% patients \\
\hline SDMT & & \\
$\quad$ Normal & 38 & 56.7 \\
$\quad$ Abnormal & 29 & 43.3 \\
PASAT & & \\
$\quad$ Normal & 39 & 58.2 \\
$\quad$ Abnormal & 28 & 41.8 \\
WLG & & \\
$\quad$ Normal & 30 & 44.8 \\
$\quad$ Abnormal & 37 & 55.2 \\
SRTD & & 79.1 \\
$\quad$ Normal & 53 & 20.9 \\
$\quad$ Abnormal & 14 & 31.3 \\
SPART & & 68.7 \\
$\quad$ Normal & 21 & \\
$\quad$ Abnormal & 46 & 64.0 \\
CDT & & \\
$\quad$ Normal & & \\
Abnormal & 63 & \\
\hline & 4 & \\
\hline
\end{tabular}

\section{RESULTS}

Sample baseline characteristics

Sixty seven patients (45 women, 22 men, ratio 2:1) with probable MS were administered and completed the BRB-N and brain MRI examination within a mean of 29 (20) days of the first appearance of neurological symptomatology. Mean age for the group was 32.3 (10.3) years. Patients' levels of education were as follows: elementary school (eight years), one patient; high school, 27 patients; academic, 39 patients. Mean neurological disability assessed by the EDSS was 2.2 (1.3). None of the patients had suffered from a psychiatric disorder that could affect cognitive performance.

\section{BRB-N performance}

Table 1 presents the results of the cognitive tests. The most frequent abnormality was found in the visual learning and recall abilities, followed by semantic verbal fluency and complex attention and concentration.

For each cognitive test a new categorical parameter was created. This categorical parameter was assigned a value of 0 ("normal") if the corresponding cognitive test value was within the normative range, ${ }^{15}$ or the value of 1 ("abnormal"), defined as one SD below the mean for normal controls. The new categorical cognitive parameters were summed per patient to reflect the number of abnormal cognitive values. Accordingly, three subgroups of patients were defined as follows: group 1, patients who had no abnormal cognitive tests; group 2, patients who had 1-2 abnormal cognitive tests; and group 3, patients who had three or more abnormal cognitive tests. Table 2 presents the distribution of patients according to the groups defined. It is of interest to note that the great

Table 2 Distribution of patients according to number of abnormal cognitive tests

\begin{tabular}{llcc}
\hline Group & $\begin{array}{l}\text { No. abnormal } \\
\text { parameters }\end{array}$ & No. patients & \% patients \\
\hline $1(n=4)$ & 0 & 13 & 6.0 \\
$2(n=36)$ & 1 & 23 & 19.4 \\
& 2 & 15 & 34.3 \\
$3(n=27)$ & 3 & 10 & 22.4 \\
& 4 & 2 & 14.9 \\
& 5 & 67 & 100.0 \\
\hline
\end{tabular}


Table 3 Distribution of patients within the composite cognitive domains

\begin{tabular}{lrc}
\hline & No. patients & $\%$ patients \\
\hline Set A: PASAT + SRT & 60 & 89.6 \\
$\quad$ Normal & 7 & 10.4 \\
$\quad$ Abnormal & & \\
Set B: SPART + CDT & & 97.0 \\
$\quad$ Normal & 2 & 3.0 \\
$\quad$ Abnormal & & 74.6 \\
Set C: SDMT + WLG & 50 & 25.4 \\
$\quad$ Normal & 17 & \\
Abnormal & & \\
\hline
\end{tabular}

majority (94\%) of patients had an abnormal score in at least one of the tests administered. The largest group of patients $(53.7 \%)$ consisted of patients who scored abnormally on one or two tests.

No correlations were found between BRB-N performance and the demographic variables age, gender, and education.

\section{Composite cognitive domains analysis}

Reasoning that cognitive domains affected in patients with probable MS may be similar to the prevalence of deficits in large community based samples of MS patients with definite diagnosis, ${ }^{22} 23$ we grouped the assessed cognitive tests into three sets: set A, information processing and learning/recent memory; set B, visuospatial abilities and executive functions; and set $\mathrm{C}$, verbal abilities and attention span. Only patients showing abnormal test scores for both tests in each set were categorised as abnormal. Table 3 displays the normal and abnormal values for the composite scores. The most frequently affected domain among subjects with probable MS was set C (25.4\% abnormal) - a composite measure of complex attention and concentration together with verbal fluency.

\section{Brain MRI and pattern of cognitive impairment}

Brain MRI measurements of volumetric disease burden in all modalities (Gd, T2, PD, and T1) did not correlate with cognitive impairment at disease onset. None of the cognitive tests showed a significant correlation with MRI disease burden. The mean MRI volume parameters were similar for all three subgroups of categorical performance of the BRB-N. Using the Mann-Whitney $U$ test no correlations were found between either the normal or abnormal values for the composite cognitive domains scores and neurological disability or brain MRI burden of disease.

\section{DISCUSSION}

Studies reporting on the frequency of disturbance of cognitive functions in MS patients show significant interpatient variability and depend on the methods used and on the type of patients examined. In up to $50 \%$ of patients in whom no cognitive disturbances are found on routine neurological examination, cognitive impairments can be elicited using sensitive and disease specific neuropsychological tests. ${ }^{24}$ Even in patients with short disease duration of less than two years, discrete impairment of cognitive function may be found in up to $60 \%$ on neuropsychological testing without impacting activities of daily living. ${ }^{25}$

To the best of our knowledge this is the first study evaluating cognitive performance in a group of patients suffering from probable MS, not treated with disease modifying agents and within the very early stage (mean: one month) of the onset of neurological symptomatology. Previous studies, although showing cognitive impairment in patients with clinically isolated syndrome of the type seen in MS, are different from our study as patients have been evaluated after longer periods since disease onset (mean 2.2 and 6.3 years respectively). ${ }^{26} 27$

Our findings in essence show that discrete cognitive impairment can be detected in up to $53.7 \%$ of patients who had abnormal scores on one (19.4\%) or two (34.3\%) neuropsychological tests. The BRB-N was designed as a screening test and is thus sensitive at the cost of specificity, and our findings should be interpreted with this in mind. This high incidence is in accordance with the findings of Zivadinov and colleagues, ${ }^{8}$ who reported that in the early phase of relapsing remitting MS (mean disease duration 3.8 years, range $1-5), 26.4 \%$ patients were judged to be cognitively impaired (abnormal cognitive scores in two tests). It is important to emphasise that despite the high rate of impaired performance on the BRB-N, not even one subject had been diagnosed as clinically suffering from dementia. Moreover, the CDT, which is a screening test for suspected dementia, failed to show abnormal results in the great majority of tested patients (94\%). It is imperative that the clinical significance of the present findings be placed in the proper context. For all patients enrolled in this study, the presence of cognitive impairment had no effect on activities of daily leaving, and impairment was not related to a dysfunction in any of the domains constituting appropriate functioning-that is, intrapersonal, social, or professional. It has been reported that cognitive impairment has no or only a weak correlation with the degree of anxiety, ${ }^{6}$ but as it may impact negatively patients cognitive performance when first confronted with the possibility that they may be suffering from MS, we have taken care to assess patients' emotional status before testing.

Only a few longitudinal studies examined the correlation between MRI abnormalities and pattern of cognitive impairment in MS. ${ }^{28} 29$

A moderately strong correlation with lesions burden on proton density weighted MRI has been reported for deficits of selective cognitive domains. ${ }^{30}$ None of these studies had included patients with probable MS or patients within the very early phase of the disease. In our study we did not find correlation between cognitive impairment and various parameters of disease burden as quantified by brain MRI. This can suggest that the inflammatory or active lesions at onset are not the direct cause of the cognitive impairment seen later in the course of MS. The relatively mild disease burden on Tl, although present in this early stage, is yet far from influencing cognitive performance.

One may postulate that the process of axonal loss, known to occur early in the disease process, ${ }^{31}$ might be associated with the current abnormal cognitive findings. This is in agreement with recent studies showing that cerebral axonal damage may begin and affect disability from the earliest stages of the disease. $^{811}$

Recent drug trials for relapsing remitting MS have shown some positive effects on neuropsychological measures. Thus, interest in defining a basic neuropsychological test battery and in deriving agreement as to the domains that need be tested in MS patients has became imperative. Fischer and colleagues $^{22}$ grouped neuropsychological measures into three sets according to the prevalence of deficits (in each set) in a large sample of MS patients, and showed that the set most commonly affected (information processing/memory) positively responded to drug intervention. In the present study we have secondarily analysed the results in line with these cognitive sets. The most common impairment found was verbal abilities/attention span followed by information processing/ memory. It is of interest to note that frequency of affected sets reported by Fischer and colleagues ${ }^{22}$ does not parallel our results. This may be related to the fact the patients described by Fischer and colleagues had longer disease duration (mean duration of disease greater than six years) compared to our population of probable MS, where mean disease duration was less than one month. This difference in the pattern of impairment according to predefined domains may reflect the balance 
between early inflammatory changes on the one hand, and the involvement of axonal pathology on the other. ${ }^{11}$

To the best of our knowledge the present study is unique and the first to show cognitive impairment in patients suffering from probable MS. Characterising the pattern of cognitive dysfunction calls for further studies supporting our findings. Nevertheless, the high rates of impairment should alert clinicians to both referrals for neuropsychological screening very early in the disease course, as well as considering treatment even when neurological impairment is minimal and brain imaging shows limited disease burden.

\section{ACKNOWLEDGEMENTS}

We thank Ms M Lavie for her assistance.

\section{Authors' affiliations}

A Achiron, Multiple Sclerosis Center, Sheba Medical Center, Tel Hashomer, Israel

Y Barak, Abarbanel Mental Health Center, Bat Yam, Israel

Competing interests: none declared

\section{REFERENCES}

1 Achiron A, Barak Y. Multiple sclerosis-from probable to definite diagnosis. A 7-year prospective study. Arch Neurol 2000;57:974-9.

2 Paty DW. Magnetic resonance in multiple sclerosis. Curr Opin Neurol Neurosurg 1993:6:202-8.

3 Filippi M, Horsfield MA, Morrissey SP, et al. Quantitative brain MRI lesion load predicts the course of clinically isolated syndromes suggestive of multiple sclerosis. Neurology 1994;44:635-41.

4 Pelosi L, Geesken JM, Holly M, et al. Working memory impairment in early multiple sclerosis. Evidence from an event-related potential study of patients with clinically isolated myelopathy. Brain 1997;120:2039-58.

5 Ron MA, Feinstein A. Multiple sclerosis and the mind. J Neurol Neurosurg Psychiatry 1992;55: 1-3.

6 Kesselring J, Klement U. Cognitive and affective disturbances in multiple sclerosis. J Neurol 2001;248: 180-3.

7 Grant I, McDonald WI, Trimble MR, et al. Deficient learning and memory in early and middle phases of multiple sclerosis. J Neurol Neurosurg Psychiatry 1984;47:250-5.

8 Zivadinov R, Sepcic J, Nasuelli D, et al A longitudinal study of brain atrophy and cognitive disturbances in the early phase of relapsing-remitting multiple sclerosis. J Neurol Neurosurg Psychiatry $2001 ; 70: 773-80$.

9 Schwid SR, Bever CT. The cost of delaying treatment in multiple sclerosis. What is lost is not regained. Neurology 2001;56:1620.

10 DeStefano N, Narayanan S, Francis GS, et al. Evidence of axonal damage in the early stages of multiple sclerosis and its relevance to disability. Arch Neurol 2001;58:65-70.
11 Biartmar C, Kidd G, Ransohoff RM. A real-time insight into disease progression and the role of axonal injury in multiple sclerosis. Arch Neurol 2001;58:37-9.

12 Poser CM, Paty DW, Scheinberg L, et al. New diagnostic criteria for multiple sclerosis: guidelines for research protocols. Ann Neurol 1983:13:227-31.

13 Fazekas F, Barkhof F, Filippi $M$, et al. The contribution of magnetic resonance imaging to the diagnosis of multiple sclerosis. Neurology 1999;53:448-56.

14 Bever CT Jr, Grattan L, Panitch HS, et al. The Brief Repeatable Battery of Neuropsychological Tests for Multiple Sclerosis: a preliminary serial study. Mult Scler 1995:1:165-9.

15 Boringa JB, Lazeron RHC, Reuling IEW, et al. The Brief Repeatable Battery of Neuropsychological Tests: normative values allow application in multiple sclerosis clinical practice. Mult Scler 2001;7:263-7.

16 Barak Y, Achiron A. The effect of interferon-beta-1B (IFN-b) on cognitive function in multiple sclerosis. Eur Neurol 2002;47:11-14.

17 Shulman KI, Gold DP, Cohen CA, et al. Clock-drawing and dementia in the community: a longitudinal study. Int J Geriatric Psychiatry 1993:8:487-96.

18 Royall DR, Mulroy AR, Chiodo LK, et al. Clock drawing is sensitive to executive control: a comparison of six methods. J Gerontol B Psychol Sci Soc Sci 1999;54:328-33.

19 Kurtzke JF. Rating neurologic impairment in multiple sclerosis: an Expanded Disability Status Scale (EDSS). Neurology 1983;33: 1444-52.

20 Barak Y, Gabbay U, Gilad R, et al. Neuropsychiatric assessment as a secondary outcome measure in a multiple sclerosis IVlg trial. Int J Psychiatry Clin Pract 1999;3:31-4

21 Stern M, Gicquel S, Miron S, et al. Three dimensional brain MR imaging of multiple sclerosis lesions: development of a new image processing software. Mult Scler 2001;7(suppl 1):S91.

22 Fischer JS, Priore RL, Jacobs LD, et al, and the Multiple Sclerosis Collaborative Research Group. Neuropsychological effects of interferon b-1 a in relapsing multiple sclerosis. Ann Neurol 2000;48:885-92.

23 Rao SM, Leo GJ, Bernardin L, et al. Cognitive dysfunction in multiple sclerosis 1. Frequency, patterns and prediction. Neurology 1991;41:685-91.

24 Brassington JC, Marh NV. Neuropsychological aspects of multiple sclerosis. Neuropsychol Rev 1998;8:43-77.

25 Lyon-Caen O, Jouvent $R$, Hauser $S$, et al. Cognitive functions in recent-onset demyelinating diseases. Arch Neurol 1986;43:1 138-41.

26 Callanan MM, Logsdail SG, Ron MA, et al. Cognitive impairment in patients with clinically isolated lesions of the type seen in multiple sclerosis. Brain 1989:112:361-74.

27 Feinstein A, Kartsounis LD, Miller DH, et al. Clinically isolated lesions of the type seen in multiple sclerosis: a cognitive psychiatric and MRI follow up study. J Neurol Neurosurg Psychiatry 1992;55:869-76.

28 Hohol MJ, Guttmann CR, Orav J, et al. Serial neuro-psychological assessment and magnetic resonance imaging analysis in multiple sclerosis. Arch Neurol 1997:54:1018-25.

29 Feinstein A, Ron M, Thompson A. A serial study of psychometric and magnetic resonance imaging in multiple sclerosis. Brain 1993; 1 16:569-602.

30 Rovaris M, Filippi M, Falautano M, et al. Relation between MR abnormalities and patterns of cognitive impairment in mutiple sclerosis. Neurology 1999:50:1601-8.

31 Trapp BD, Peterson J, Ransohoff RM, et al. Axonal transection in the lesions of multiple sclerosis. N Engl J Med 1998;338:278-85. 